\title{
DESULFURACIÓN DE RELAVE MEDIANTE LA FLOTACIÓN DE SULFUROS DE HIERRO
}

\author{
L. VALDERRAMA*, B. ZAZZALI, J. CHAMORRO y M. SANTANDER \\ Departamento de Metalurgia - Universidad de Atacama \\ luis.valderrama@uda.cl*
}

Manuscrito recibido en noviembre / 2015 y aceptado en diciembre / 2015

DOI: $10.15628 /$ holos.2015.3773

\section{RESUMEN}

En Chile la minería se desarrolla principalmente en la concentración de cobre, molibdeno, oro y plata, mediante el proceso de flotación. Este genera grandes tonelajes de relaves que contienen diversas minerales, principalmente pirita. Dado que estos minerales se depositan en los tranques, estos pueden generar aguas ácidas; se propone como alternativa la desulfuración de los súlfuros por flotación. En una primera parte se estudió la flotabilidad de la pirita en un tubo Hallimond, utilizando como colector AP 404. Luego se realizaron pruebas de flotación a nivel de laboratorio en celda Denver D-12, utilizando pulpa de relave fresco de la etapa rougher de un circuito de flotación de cobre, determinando el pH óptimo y dosificación de colector y espumantes. Pruebas de cinética de flotación permitieron determinar el tiempo óptimo de flotación tanto para la etapa rougher como para la etapa scavenger. Se concluye que la flotación de los relaves sea una técnicamente factible su desulfuración, obteniendo relaves que contienen $0,08 \%$ de pirita.

PALABRAS CLAVE: desulfuración, relave, piritas, flotación.

\section{DESULPHURIZATION BY FLOTATION TAILINGS OF IRON SULFIDES}

\begin{abstract}
In Chile mining takes place mainly in the concentration of copper, molybdenum, gold and silver by flotation process. This generates large tonnages of tailings containing various minerals, primarily pyrite. Since these minerals can generate acid waters that are deposited in tailings, it is proposed as an alternative the sulphides desulfurization by flotation. In the first part the pyrite flotability in a Hallimond tube was studied, using as
\end{abstract}

collector AP 404. Then laboratory flotation tests at a Denver D-12 cell were performed using fresh pulp tailings from a rougher stage of a copper flotation circuit, determining the optimum $\mathrm{pH}$ and dosage of collector and foaming. Flotation kinetic tests allowed to determine the optimal flotation time for both rougher stage and scavenger stage. We conclude that the tailings flotation makes technically desulfurization feasible, obtaining tailings with a pyrite contents of $0.08 \%$.

KEYWORDS: desulphurization , tailings , pyrite , flotation 


\section{INTRODUCCIÓN}

La minería es una actividad que produce gran deterioro al medio ambiente, cuyo efecto nocivo se refleja en la degradación de vastas extensiones de terreno en los que se depositan grandes volúmenes de residuos sólidos y la consecuente generación de aguas ácidas, Newman, et al. (2001). Este fenómeno se observa reiteradamente en nuestro país puesto que es uno de los principales productores de cobre a partir de minerales sulfurados y oxidados. El ejemplo más palpable lo tenemos en la Región de Atacama, donde la pequeña, mediana y gran minería producen grandes volúmenes de relaves.

La generación de aguas ácidas en los tranques de relaves, como resultado de la oxidación de los sulfuros residuales, es un problema ambiental importante para la minería. Diferentes impactos ambientales han sido descrito por Ripley et al., (1996) y Gray, (1997).

En Canadá se están empleando diferentes técnicas para reducir la generación de aguas ácidas, entre las que se encuentran limitar el contenido de uno de los componentes (agua, oxígeno, o sulfuros), recubrimientos con materiales sintéticos de baja conductividad, usos de bentonita, etc, Bienvenu y Dufour, (1996); Harris, et al. (2003).

La eliminación del azufre contenido en los relaves es otra forma de limitar la producción de aguas ácidas. Investigaciones sobre esta técnica han sido reportados por Humber, (1995); Bussière et al, (2004), Benzaazoua et al., (2000). Esta técnica consiste en enriquecer el sulfuro -contenido en el relave- en un concentrado de ley comercial mediante flotación, de esta forma es posible reducir sustancialmente el volumen de agua ácida generado por estos relaves.

Según el Servicio Nacional de Geología y Minería, SERNAGEOMIN, en la Región de Atacama existen 164 depósitos de relaves de los cuales 55 se encuentran en actividad, 18 están paralizados (no activos) y 91 en la calidad de abandonados. En consideración a su tamaño, cercanía a centros poblados y cursos de aguas principales, se han identificado nueve depósitos con especial interés de peligrosidad por su ubicación frente a las lluvias.

La mayoría de los relaves contiene una gran cantidad de pirita residual, sulfuros metálicos y reactivos de flotación, los que al no estabilizarse adecuadamente con el tiempo podrían causar graves problemas ecológicos.

La planta Manuel Antonio Matta de la Empresa Nacional de Minería, inició sus operaciones en el año 1974, por lo cual en la actualidad tiene un tonelaje de relaves almacenado de 30.000.000 toneladas. La presencia de hierro en magnetita y pirita, $\mathrm{Ti}, \mathrm{Cu}, \mathrm{Au}$ y $\mathrm{Ag}$ en los relaves, hacen que su beneficio sea atractivo, Valderrama, et al. (2011).

El tratamiento de los relaves puede ser considerado como una alternativa rentable cuando contienen cantidades relativamente altas de metales. Por otra parte, dado que los relaves no implican costos de extracción, chancado, molienda y procesamiento, su retratamiento permite generar un flujo de caja positivo.

En este trabajo experimental se evalúa la factibilidad técnica de reducir, mediante el proceso de flotación, la cantidad de pirita que contiene estos relaves, y con el concentrado de pirita obtenido pueda ser vendido para la producción de ácido sulfúrico o la fabricación de ácido sulfhídrico. 


\section{PARTE EXPERIMENTAL}

\subsection{Obtención de Muestras}

El estudio de micro flotación fue realizado con pirita de alto grado de pureza, la que se seleccionó a mano desde un mineral que contenía gran cantidad de pirita visualmente pura.

Las muestras para las pruebas de flotación fueron recolectadas del relave fresco producido, al momento de beneficiarse el mineral de cobre.

\subsection{Reactivos}

En las pruebas de flotación se utilizó como colector di sec butil-ditiofosfato de sodio (AP 404), comercializado por Cytec, y como espumante metil isobutil carbinol (MIBC) y éter poli propilen glicol (D-250), además de ácido sulfúrico, ácido clorhídrico, hidróxido de sodio y cal para ajustar el pH.

\subsection{Equipos}

Las pruebas de micro flotación fueron realizadas en un tubo Hallimond de $200 \mathrm{~cm}^{3}$ de capacidad, usando nitrógeno presurizado.

Las pruebas de flotación de laboratorio fueron realizadas en una celda Denver D-12 de 2,66 litros de capacidad.

La distribución de tamaños de las partículas fue determinada mediante en una serie de tamices desde la malla 70, 100, 140, 200, 270 y 400 de la serie A.S.T.M.

El peso específico del material sólido se determinó por medio de la técnica del picnómetro.

\subsection{Metodología}

La muestra de relave fue tomada en la descarga del circuito rougher, utilizando un cortador de muestras de $1 / 2$ " de abertura y con capacidad de 3 litros de volumen, descargando en forma inmediata en la celda de flotación.

Las pruebas de micro flotación tuvieron como objetivo verificar la flotabilidad de la pirita con el colector AP 404, en el rango de $\mathrm{pH}$ de 5-11, además del efecto de los ácidos $\mathrm{H}_{2} \mathrm{SO}_{4}$ y $\mathrm{HCl}$, utilizados como modificadores del $\mathrm{pH}$. Se realizaron pruebas en las que se modificó la concentración de colector $\left(1 \times 10^{-4} \mathrm{M}\right.$ y $\left.1 \times 10^{-3} \mathrm{M}\right)$ y del modificador de $\mathrm{pH}$.

Las pruebas de flotación fueron realizadas en una celda Denver D-12 de laboratorio, con el objetivo de identificar el pH óptimo de la flotación, como también la dosificación óptima del colector A-404. Posteriormente se llevaron a cabo pruebas cinéticas de flotación para las etapas rougher y scavenger. 


\section{RESULTADOS Y DISCUSIONES}

El análisis químico de la pirita, seleccionada visualmente a mano desde el mineral de cobre chancado a $-1 / 4{ }^{\prime \prime}$, reportó una ley de $98,7 \%$, cuya pureza es suficiente para las pruebas de flotabilidad en el tubo Hallimond.

La tabla 1 muestra el análisis químico de la muestra de relave obtenido y además del análisis químico realizado a las mallas $70,100,140,200,270,400$ y -400 A.S.T.M.

Tabla 1. Análisis granulométrico y químico del relave.

\begin{tabular}{|c|c|c|}
\hline Muestra & Peso (\%) & Ley Pirita (\%) \\
\hline 70 & 9,8 & 1,2 \\
\hline 100 & 16,4 & 1,6 \\
\hline 140 & 3,3 & 2,8 \\
\hline 200 & 12,9 & 3,2 \\
\hline 270 & 9,3 & 3,3 \\
\hline 400 & 9,7 & 3,8 \\
\hline-400 & 38,6 & 4,2 \\
\hline Total & 100 & 3,2 \\
\hline
\end{tabular}

La muestra obtenida a partir de la pulpa de relave indica una ley de pirita de 3,2\%, constatándose que existe una homogeneidad de leyes de pirita entre las mallas 200 a la -400, con una ley promedio aproximada de $3,6 \%$. La tabla también muestra que el porcentaje pasante en la malla 200 es 57,6\%, razón por la cual se deduce que el material no tiene una molienda adecuada para todas las etapas de flotación.

El análisis mineralógico indicó la presencia de especies metálicas tales como magnetita, hematita, pirita y calcopirita, siendo la magnetita la especie predominante. Respecto de la pirita, el relave que la contiene posee partículas con tamaños de $4 \mu \mathrm{m}$ hasta $150 \mu \mathrm{m}$, observándose que las partículas que se encuentran ocluidas están principalmente asociadas a partículas mayores de no metálicos, tales como sílice, y en menor grado en minerales metálicos, tales como magnetita.

El valor del peso específico del sólido obtenido mediante la técnica del picnómetro fue de 2,98 Kg/L.

\subsection{Estudio de micro flotación en tubo Hallimond.}

Se realizaron pruebas de micro flotación, en donde se hizo un barrido del $\mathrm{pH}$ desde 11 hasta 5 con dos tipos de ácido $\left(\mathrm{HCl}\right.$ y $\left.\mathrm{H}_{2} \mathrm{SO}_{4}\right)$ y se emplearon dosificaciones de colector AP 404 de $1 \times 10^{-4} \mathrm{y}$ $1 \times 10^{-3}$ molar, cuyos resultados obtenidos se presentan en la figura 1.

De la figura se desprende que la pirita posee una excelente flotabilidad en el rango de $\mathrm{pH}$ desde 5 hasta 8 , con valores sobre el $80 \%$. Para las pruebas en el que el pH se ajustó desde 9 hasta 11, la flotabilidad de la pirita comienza a disminuir completamente a valores menores de $50 \%$ y menores, aunque la prueba 4 tiene una flotabilidad aceptable a pH 9. Se ve además que la dosis de colector tiene poco efecto en la flotabilidad a pH en el rango de 10 a 11; sólo cuando la pulpa se ajusta a pH iguales o menores a 8 el colector es capaz de adsorberse e hidrofobizar la superficie de la pirita. 


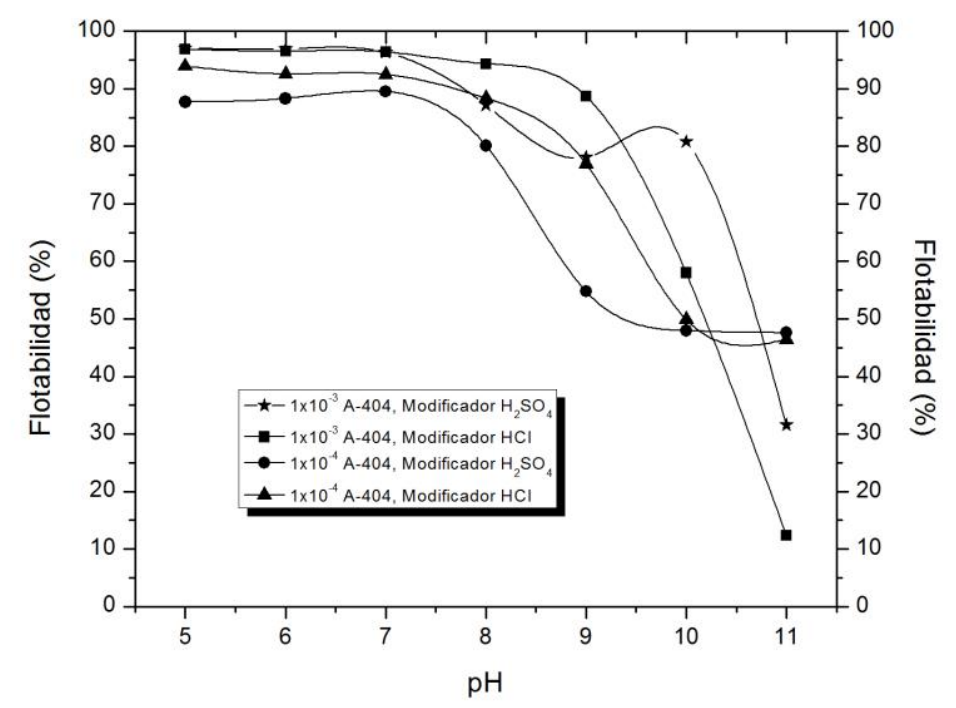

Figura 1. Flotabilidad de la pirita utilizando colector A-404.

\subsection{Pruebas de flotación para determinar el pH}

En estas pruebas se varió el pH de trabajo desde 5,3 hasta 7,9, rango determinado por las pruebas en el tubo Hallimond. La figura 2 muestra el gráfico de $\mathrm{pH}$ v/s recuperación y $\mathrm{pH} \mathrm{v} / \mathrm{s}$ ley de pirita.

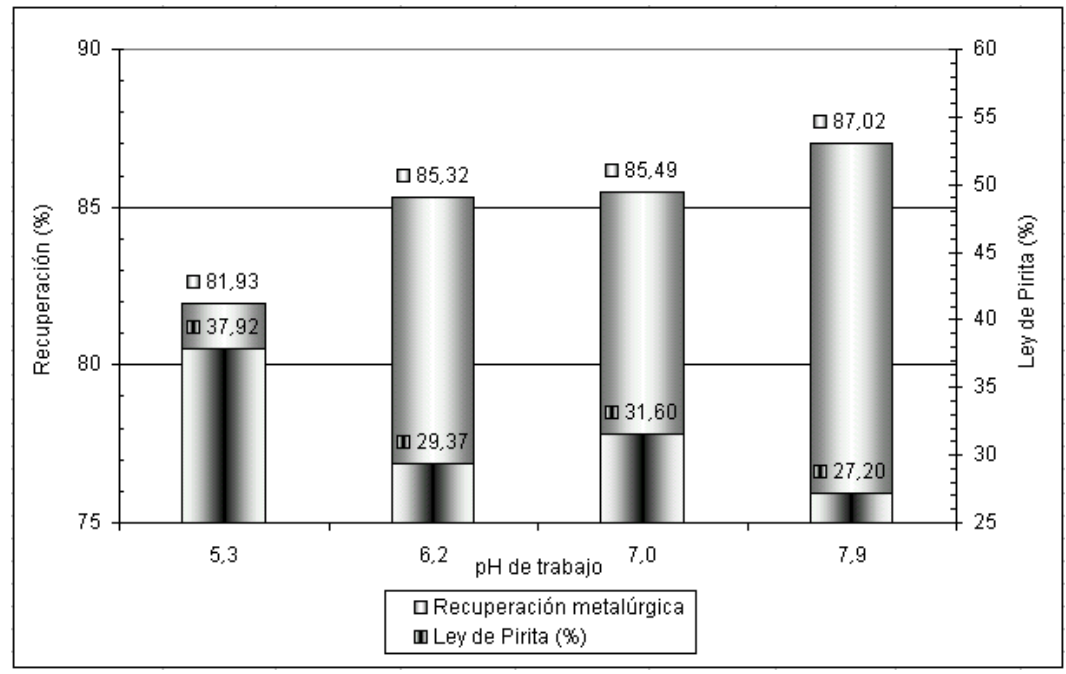

Figura 2. Gráfico de recuperación metalúrgica y ley de pirita en el concentrado.

De acuerdo a la figura 2, la mejor recuperación metalúrgica se alcanza en la prueba realizada a pH 7,9 (87,02\%) y también la menor ley de concentrado $(27,11 \%$ de pirita), la menor recuperación metalúrgica se reporta en la prueba realizada a $\mathrm{pH} 5,3(81,93 \%)$ y la mayor ley de concentrado (37,92\% de pirita). La diferencia entre la mayor y la menor recuperación alcanza aproximadamente 5 puntos porcentuales.

La figura 3 muestra el gráfico de $\mathrm{pH}$ v/s recuperación y $\mathrm{pH}$ v/s ley de pirita para la pruebas usando un $\mathrm{pH} 8$, variando la dosis de colector entre 10 hasta $50 \mathrm{~g} / \mathrm{t}$. 
En dicha figura se observa que la recuperación metalúrgica de pirita alcanza valores de $84,73 \%, 87,02 \%$ y $81,30 \%$, respectivamente, con el máximo de recuperación en la prueba realizada con la dosificación de colector de $30 \mathrm{~g} / \mathrm{t}$ (87,02\%). Se aprecia también que una dosificación de colector igual o mayor a $40 \mathrm{~g} / \mathrm{t}$ no reportaría una mayor recuperación metalúrgica, como tampoco una ley del concentrado mayor que las conseguidas con la prueba realizada con la dosificación de $30 \mathrm{~g} / \mathrm{t}$ de colector, ya que se aprecia un franco descenso de los índices estudiados al aumentar las dosis de colector, una dosificación bajo $20 \mathrm{~g} / \mathrm{t}$ se considera insuficiente.

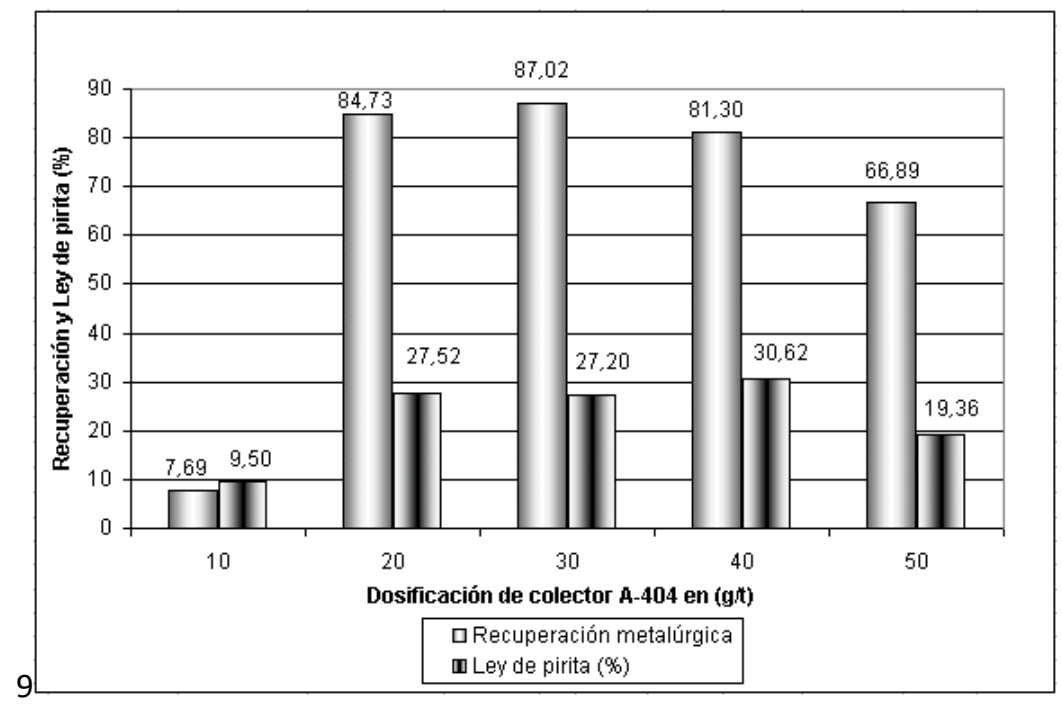

Figura 3. Gráfico de recuperación metalúrgica y ley de pirita del concentrado.

\subsection{Cinéticas de flotación Rougher .}

Los resultados obtenidos en la prueba de cinéticas de flotación rougher se muestran en la tabla 2 .

Tabla 2. Balance metalúrgico de prueba cinética de rougher.

\begin{tabular}{ccccc}
\hline $\begin{array}{c}\text { Tiempo } \\
\text { (min) }\end{array}$ & $\begin{array}{c}\text { Peso } \\
(\mathbf{g})\end{array}$ & $\begin{array}{c}\text { Ley } \mathbf{P y} \\
\mathbf{( \% )}\end{array}$ & $\begin{array}{c}\mathbf{R}_{\mathbf{P}} \\
\mathbf{( \% )}\end{array}$ & $\begin{array}{c}\mathbf{R}_{\mathbf{M}} \\
\mathbf{( \% )}\end{array}$ \\
\hline 1 & 52,0 & 62,7 & 4,1 & 65,9 \\
\hline 2 & 16,3 & 50,2 & 5,4 & 81,5 \\
\hline 3 & 7,5 & 36,1 & 6,0 & 87,0 \\
\hline 5 & 8,0 & 22,9 & 6,6 & 90,6 \\
\hline 7 & 4,8 & 16,7 & 7,0 & 92,2 \\
\hline 10 & 5,0 & 11,2 & 7,4 & 93,4 \\
\hline 15 & 6,7 & 7,8 & 7,9 & 94,3 \\
\hline Relave & $1.167,7$ & 0,2 & 92,1 & 4,7 \\
\hline Alimentación & $1.268,0$ & 3,9 & 100,0 & 100,0 \\
\hline
\end{tabular}

Se puede observar que la recuperación metalúrgica no se incrementa sustancialmente después de 5 minutos de flotación, pudiendo definirse éste último como tiempo de flotación, con la salvedad que la ley parcial de pirita en el concentrado es de $22,9 \%$, valor que fue considerado alto para definir a los 5 minutos como tiempo de flotación, por eso fue determinado en 10 minutos el tiempo de flotación donde la ley parcial tiene el valor de $11,2 \%$ con una recuperación de 93,4\%, de 
esta forma se obtendría un relave con una ley de 0,24\% de pirita, el que podría ser sometido a una etapa de flotación scavenger para obtener relave con una menor ley de pirita.

\subsection{Cinética de flotación scavenger}

Los resultados de la prueba de cinética de flotación scavenger se muestran en la tabla 3, que presenta el balance metalúrgico de la prueba cinética.

Tabla 3. Balance metalúrgico de la prueba cinética de flotación scavenger.

\begin{tabular}{ccccc}
\hline $\begin{array}{c}\text { Tiempo } \\
\text { (min) }\end{array}$ & $\begin{array}{c}\text { Peso } \\
(\mathbf{g})\end{array}$ & $\begin{array}{c}\text { Ley } \mathbf{P y} \\
(\mathbf{\%})\end{array}$ & $\begin{array}{c}\mathbf{R}_{\mathbf{P}} \\
(\mathbf{\%})\end{array}$ & $\begin{array}{c}\mathbf{R}_{\mathbf{M}} \\
(\mathbf{\%})\end{array}$ \\
\hline 1 & 2,7 & 14,3 & 0,24 & 17,4 \\
\hline 5 & 5,1 & 7,8 & 0,70 & 35,3 \\
\hline 7 & 9,5 & 5,6 & 1,6 & 58,9 \\
\hline 10 & 9,0 & 0,6 & 2,3 & 61,2 \\
\hline Relave & $1.082,7$ & 0,08 & 96,7 & 38,8 \\
\hline Alimentación & $1.120,0$ & 0,2 & 2,24 & 100,0 \\
\hline
\end{tabular}

Se aprecia claramente en esta tabla que hasta el minuto 7 la recuperación de pirita aumenta de forma importante, donde se obtiene una recuperación final de $58,9 \%$ con una ley de pirita en el concentrado de 5,6\%. Desde el minuto 7 hasta el minuto 10 la recuperación no se incrementa significativamente. Dado que se quiere reducir el contenido de azufre en el relave al mínimo posible, se propone que el tiempo de flotación para la etapa scavenger sea de 10 minutos, lo que produciría un relave con una ley de $0,08 \%$ de pirita.

\section{CONCLUSIONES}

En base a los resultados del estudio se puede concluir lo siguiente:

Las pruebas realizadas en el tubo Hallimond muestran claramente que la mejor flotabilidad de la pirita con colector A-404 se encuentra en el rango de $\mathrm{pH}$ desde 5 a 8, condiciones que permiten reducir la pirita en un porcentaje superior al $80 \%$.

Las pruebas cinéticas de flotación permiten fijar un tiempo de flotación de 10 minutos para la etapa rougher, con una recuperación metalúrgica de 93,4\%, tiempo sobre el cual se debe incluir una etapa scavenger para obtener un relave con un contenido de pirita menor a 0,08\%.

De acuerdo a las consideraciones anteriores, este estudio muestra que es posible generar un impacto ambiental positivo si se logra disminuir una gran parte de la pirita destinada a ser depositada en los relaves; en caso contrario, con el paso de los años su presencia en los relaves creará la condiciones necesarias para la producción de aguas ácidas.

\section{BIBLIOGRAFIA}

1. BenzaAZOUA, M., Bussière, B., KONGOlO, M., McLAUghliN, J. and MARION, P. 
Environmental desulphurization of four Canadian mine tailings using froth flotation. International Journal of Mineral Processing, $6: 57-74.2000$.

2. BIENVENU, L. and DUFOUR, C. Restauration des sites Somex et Lemoine. Colloque sur le Programme de neutralisation des eaux de drainage dans l'environnement minier, RouynNoranda, vol. 1: 185-195. 1996

3. BUSSIÈRE, B., BENZAAZOUA, M., AUBERTIN, M. and MBONIMPA M. Performance of covers made of low sulphide tailings to prevent acid mine drainage. Journal of Environmental Geology. vol 45 : 609-622. (2004).

4. GRAY, N.F. Environmental impact and remediation of acid mine drainage: a management problem. Environmental Geology, 30: 62-71. 1997.

5. HARRIS, D.L., LOTTERMOSER, B.G., \& DUCHESNE, J., (2003). Ephemeral Acid Mine Drainage At The Montalbion Silver Mine, North Queensland. School of Earth Science, James Cook University. Australia, 50(5), 797-809.

6. http://www.sernageomin.cl/pdf/mineria/seguridad/estudios/Catastro-depositos-relave-de-Chilejulio2015.pdf

7. HUMBER, A.J. Separation of sulphide minerals from mill tailings, Sudbury'95, Conference on Mining and the Environment, vol. 1 : 149-158. 1995

8. NEWMAN, P., WHITE, R., \& CADDEN, A. (2001). Paste - the Future of Tailings Disposal? International Conference on Mining and the Environment, Skelleftea, Sweden. 594-603.

9. RIPLEY, E.A., REDMANN, R.E. and CROWDER, A.A. Environmental Effects of Mining, St. Lucie Press. 1996.

10. VAlderRama, L., SANTANDER, M., PAVEZ, O., GUeVARA, M., CASTILlO, C., HUMERES, P. Caracterización de pirita contenidas en relaves de cobre. Revista de la Facultad de Ingeniería de la Universidad de Atacama, 2011. 